\title{
Visual Mementos: Reflecting Memories with Personal Data
}

\author{
Alice Thudt, Dominikus Baur, Samuel Huron, and Sheelagh Carpendale
}

\begin{abstract}
In this paper we discuss the creation of visual mementos as a new application area for visualization. We define visual mementos as visualizations of personally relevant data for the purpose of reminiscing, and sharing of life experiences. Today more people collect digital information about their life than ever before. The shift from physical to digital archives poses new challenges and opportunities for self-reflection and self-representation. Drawing on research on autobiographical memory and on the role of artifacts in reminiscing, we identified design challenges for visual mementos: mapping data to evoke familiarity, expressing subjectivity, and obscuring sensitive details for sharing. Visual mementos can make use of the known strengths of visualization in revealing patterns to show the familiar instead of the unexpected, and extend representational mappings beyond the objective to include the more subjective. To understand whether people's subjective views on their past can be reflected in a visual representation, we developed, deployed and studied a technology probe that exemplifies our concept of visual mementos. Our results show how reminiscing has been supported and reveal promising new directions for self-reflection and sharing through visual mementos of personal experiences.
\end{abstract}

Index Terms—Visual Memento, Memories, Personal Visualization, Movement Data, World Wide Web

\section{INTRODUCTION}

Keeping mementos, creating pictures, diaries and other autobiographical representations of experiences are important practices for preserving precious memories. The current shift from physical mementos to digital collections is impacting people's practice of self-reflection and self-representation $[43,47,46,62]$. Today a large variety of tools support digital capture of different aspects of people's lives. However, the temptation to capture as much as possible results in vast collections scattered over disparate sources. This huge amount of data can lead to an emotional alienation from digital collections and complicate people's construction of meaning and connection to their past. These issues have made the creation and exploration of digital mementos an active research topic in Human Computer Interaction and Ubiquitous Computing (e.g [26, 33, 43, 46, 47, 51, 63]). However, thus far, this area has been under-explored in visualization. In this paper, we propose visual mementos as a new application area for visualization and study reactions to a technology probe for creating visual mementos.

Extensive research in psychology has explored the act of reminiscing (the "enjoyable recollection of past events" [55]), and the construction of autobiographical memories [8, 17, 15, 22, 67]. This research suggests that memories are not just an objective capture of our past. Rather, they are constantly redefined through reminiscing. This constant reinterpretation makes autobiographical memories inherently subjective. The subjectivity of our memories poses interesting challenges for applying visualizations to the domain of personal mementos. While visualizations commonly aim for objective visual mappings, visual mementos explore the inclusion of adaptable and subjective representational mappings to include more personal perspectives. One important strength of visualization is revealing unknown patterns in abstract data to discover insights. In the case of visual mementos, this strength can be used for revealing familiarity to trigger memories. The aspect of privacy poses another challenge when sharing visual mementos: being able to obscure sensitive details may be more appropriate than showing data as detailed as possible.

In order to assess visual mementos in use, we developed and deployed a technology probe for the web that allows people to load and visualize personal movement data, adjust their representation, and

- A. Thudt, S. Carpendale and S. Huron are with the University of Calgary. S. Huron is also with IRI.Emails: alice.thudt@gmail.com, samuel.huron@cybunk.com, sheelagh@ucalgary.ca

- D. Baur is an independent researcher.Email: do@minik.us.

Manuscript received 31 Mar. 2015; accepted 1 Aug. 2015; date of publication xx Aug. 2015; date of current version 25 Oct. 2015. For information on obtaining reprints of this article, please send e-mailto:tvcg@computer.org. share it. This system carefully incorporates factors designed to allow assessment of the identified challenges and allowed us to explore whether visual mementos can be created by a system. In developing our system, visits ${ }^{1}$, we make use of the map-timeline representation [59] because its use of the semantic structure of autobiographical memories fits with our intention of showing familiar patterns. To express subjectivity, our visual memento augments the map-timeline by integrating pictures and providing features for defining personally significant time periods and for aggregating logs into semantic entities. Our system further implements adjusting privacy settings as a means for hiding sensitive details by adjusting the aggregation level when sharing. We deployed this system online to understand people's use of visual mementos. Our results show that participants appreciated the visual representation and found their memories well-reflected. Participants used visits for personal reminiscing as well as for sharing of their experiences with others. The main contributions of this paper are:

1. Defining visual mementos as a new application area for visualization by outlining their purposes and design challenges based on a broad review of previous literature;

2. Investigating the concept of visual mementos by creating an operational web service that enables people to build a visual memento from their own personal movement data;

3. Exploring the potential of visual mementos to support reminiscing by studying people's reactions to our technology probe.

\section{Related Work}

This work on visual mementos emerges at the intersection of research on personal visualization and digital mementos. Digital mementos are an active research interest in domains such as Human Computer Interaction and Ubiquitous Computing. Although digital memento systems support the exploration of large personal data collections, only few systems consider the use of visualization. We introduce visual mementos as a promising domain for visualization by providing an extensive literature review of previous work from related fields.

\subsection{Digital Mementos}

Digital mementos have been discussed under different themes. Lifelogging software addresses capture and retrieval of data about one's life to augment human memory. Empirical investigations of digital and physical mementos aim to understand people's practices and inform technological support. Finally, systems for digital mementos help to create, explore and share these artifacts.

The research area of lifelogging and personal informatics aims to provide tools that help to capture data about one's personal life in order to keep a record of the past. The vision of lifelogging research is to

\footnotetext{
${ }^{1}$ http://v.isits.in/
} 
enable "total recall" of our lives [7] by capturing vast amounts of data and providing people with access to the collected material $[7,20]$ or quantifying aspects of one's life [14] to derive insights from the data. This vision was later criticized by [52], saying that the ultimate goal should be to derive meaning from the collected data. The challenges we identify for visual mementos (Section 3) also focus on supporting more meaningful connections with captured datasets. We specifically explore whether visualizations of automatically collected data can adequately reflect an individual's personal perception of their past.

Numerous qualitative studies investigate theories about autobiographical memories [63], and people's relationships with digital archives and mementos. The goal of these studies is to inform the design of technology for reminiscing and to explore the impact of digital personal archives on people's practices with their collections $[28,33,42,43]$. Some research has examined the use of physical mementos in order to identify challenges for digital mementos $[33,47,46]$. Results show that mementos have two important purposes: they can be used as symbols of past experiences that trigger memories[19, 46, 47] and stimulate conversations about the past with others to form social bonds $[67,46,5]$. The findings of this research inspired the design of digital systems such as the Living Memory Box that supports capture of speech and video to store family mementos [54]. Other systems support organizing collections of mementos. MemoryLane, for instance, allows people to spatially arrange icons of digital mementos in different views [32]. These systems are more concerned with capture and curation rather than the creation of artifacts from digital datasets. We adopt the purposes of reminiscing and social sharing for visual mementos as representations of personal data.

The aspect of sharing and joint reminiscing with digital mementos has been mostly explored in the context of digital photo collections. Investigations include remote and co-located [26], as well as online photo sharing [50]. Many systems address scenarios where photos are explored in groups for joint reminiscing, e.g. on tabletop displays $[30,53]$. Another stream of research aims to seamlessly integrate these digital mementos into the domestic environment $[2,41,45]$. This research emphasizes that sharing of memories with others is an important function of mementos. Our research extends this stream by exploring the use of visualization to support sharing of mementos.

\subsection{Personal Visualization}

Information visualization systems have often focused on supporting domain experts in professional settings. Recently, researchers have highlighted new application subdomains closer to the topic of mementos: casual visualization [49] and personal visualization [31].

Huang et al. provide a broad survey of personal visualization systems for data analysis by non-experts in everyday life [31]. These systems visualize personal data from a wide range of sources (e.g. from sensors for one's sleep cycle [4], movement [32, 59], logs of computer usage [3], music listening histories [5, 6], as well as communication logs $[29,66])$ and about different aspects of people's lives (e.g. one's health, nutrition, social life, spending behaviour, productivity and ecological footprint). Personal visualizations of this wide variety of data are often used to inform behaviour change that can improve aspects of people's lives through personal analytics [31]. Supporting personal reflection is often mentioned as a goal in personal visualizations (e.g. [37]), but few systems support self-reflection in the context of reminiscing (see Section 2.3). One popular area of personal visualizations is movement data. In contrast to the visual analytics approach of deriving actionable insight from this type of data (e.g. [1]), personal visualizations aim at personal meaning. Cyclical patterns (e.g. [35]), displaying sections of interest (e.g. [24]), artistic exploration (e.g. LiquiData $^{2}$ ) or emphasizing activity (e.g. Moves ${ }^{3}$, Move-O-Scope ${ }^{4}$ ), enable personal understanding. These systems, however, usually focus on analysis. We focus on a specific type of personal visualization, in which the main goal is to reflect on one's past as part of reminiscing.

\footnotetext{
${ }^{2} \mathrm{http}: / /$ www.liquidata.org/

${ }^{3} \mathrm{http}: / /$ moves-app.com

${ }^{4}$ https://move-o-scope.halftone.co/
}

In their survey, Huang et al. also describe sharing as a challenge for personal visualizations [31]. Sharing is beneficial in the context of representations that reflect personal memories [48, 58], but also to compare goals and personal progress with others [13, 38]. In these scenarios, privacy becomes an issue as it may not be desirable to share all aspects of the data. An example of a visualization used to maintain privacy is UbiFit [16], in which the goal for using a metaphorical representation was to create an engaging view while also using this representation to make the data difficult for others to interpret. Thudt et al. [60] highlighted this challenge and hinted at the potential to use visualization for customizable privacy. In this paper, we explore how visualization can be used to obscure certain data aspects when sharing visual mementos with different audiences.

\subsection{Visualizations for Reminiscing}

Few visualizations of personal data have focused on the purpose of reminiscing. As described earlier, reminiscing encompasses the recall of personal experiences and the communication of these experiences to others. We can classify visualization systems by their type of visual mapping (standard techniques or specific representations) and by the purpose they support (recall or sharing of past experiences).

Most existing visualization systems for reminiscing make use of standard techniques such as maps, bar and line charts, and timelines etc $[3,27,32,44,58]$. SnapTracks [32] uses a map to show the spatial distribution of automatically collected Sensecam [51] pictures to investigate how lifelogs can be used to reflect on the past. A similar representation is used by Memory Book [44] and in the Autographer System ${ }^{5}$. AppInsight [3] uses bar charts to show computer usage logs for reminiscing. Muse [29] uses a stacked graph to show communication logs. While initially designed as a system for personal recall, the MyLifeBits system was later used and studied as an authoring tool for storytelling [27]. It uses a map and photos and shows data from sensor logs. Thiry et al. [58] investigate how people can use timelines to create an artifact that reflects their past and helps to construct personal meaning. This system can also be used for sharing purposes. While all of these examples use visualization for the purpose of reminiscing, the visual mapping and the interaction concept are not geared specifically to the creation of a memento. In this paper, we discuss the challenges for creating visual mappings that support reminiscing.

Few examples include a specific visual mapping to recall and share memories. Artifacts of the Presence Era captures and shows the activity in a museum space. The authors describe their goals as "instead of creating a visualization tool for data analysis, we chose to produce a piece that functions as a souvenir of a particular time and place" [65]. While this system is not designed for personal reminiscing, it serves as an "evocative souvenir" of public activity for collective exploration. The social function is further explored in arcs.fm which visualizes listening histories of two people in order to encourage music discussions [5]. Donath et al. coined the term "data portraits" and describe them as "subjective renderings" for "self-representation" [21]. They show the personality of an individual [66] or a group [69]). This work raises the issue of the subjectivity of visual mapping for communicating personal identity. We include the challenge of subjective representations in our discussion of visual mementos.

Other visualizations for reminiscing have focused on supporting personal recall. In a study of The Mail, Viegas et al [66] found that participants "appreciated having expectations [...] confirmed by the visualization". Tat and Carpendale [57] identified similar reactions to a visualization of chat histories. Some systems that were originally designed for personal recollection and reminiscing were appropriated by participants for communicating stories. Although LastHistory [6] was designed for the analysis of listening histories and reminiscing, participants were interested in annotating and sharing their visualizations, a feature that was not integrated. Viegas et al. [64] present similar findings from their study of two visualizations of communication records. From this research, we identify two lessons for the design of visual mementos: first, people appreciate recognizing familiar aspects in the

${ }^{5}$ http://www.autographer.com/ 
data and second, even if a visualization is not made for sharing, people may use it as a memento and share it with others.

This previous research shows that revealing familiar patterns and expressing subjectivity are important challenges in the context of reminiscing with visualizations. It also suggests that visualization can help to create artifacts for social sharing of mementos. We draw from the challenges identified in this previous research in our discussion of visual mementos.

\section{Using Visualization to Create Mementos}

Mementos are objects kept as a reminder of people, places and experiences. They carry personal symbolic meaning and can be used to privately reflect on the past or share memories [19, 47, 67]. Despite their prevalence and the growing interest in mementos in other disciplines, the application area of personal mementos has so far been under-explored in visualization. We define visual mementos as visualizations of personally relevant data for the purpose of reminiscing, and sharing of experiences. Our presentation of the challenges of this new application area for visualization, builds on research on autobiographical memory, studies on the use of mementos [8, 11, 15, 19, 40, 46, 47], as well as the related literature. We present the purposes of mementos, motivate the use of visualization, and discuss and compare visual mementos to visualizations in analytic scenarios.

\subsection{Purpose of Visual Mementos}

The purposes of visual mementos are similar to those of physical mementos such as photo albums, travel journals, and souvenirs: they support personal recall of past events and sharing of experiences.

P1: Reminiscing is defined as "indulging in enjoyable recollection of past events" [55]. Research on autobiographical memory suggests that reminiscing helps people form an identity by constantly reconstructing their life story $[8,12,17]$. Mementos can be used to trigger memories and lead into episodes of reminiscing [19, 46, 47]. They are often used as symbols for significant experiences which define one's identity $[47,67]$. Visualizations of personally relevant data can be used as mementos to stimulate reminiscing and personal insight $[6,64]$ by showing aspects and patterns of people's lives.

P2: SHARING OF PERSONAL EXPERIENCES helps people make social connections by revealing stories about their lives. Autobiographical narratives support social bonding [15, 67]. Mementos are often used as a prop for storytelling $[46,67]$ and to start conversations about experiences. Visual mementos draw on the potential of visualization for communication. However, to allow sharing of personal and sensitive information they have to address the issue of privacy.

\subsection{Challenges for Visual Mementos}

Our discussion of the use of visualization to create visual mementos draws primarily upon the following three aspects of visualization: mapping data to visual representations; revealing patterns in the data; and being precise about all data details. However, upon application specific challenges arise. While visualization usually aims for objective visual representations, with visual mementos, we must consider subjectivity. The common visualization goal to both "detect the expected and discover the unexpected" [18], changes in visual mementos to evoke familiarity because through familiarity reminiscing may happen. Additionally, flexible filtering of sensitive details may be useful for addressing privacy when sharing visual mementos with different audiences, e.g. with one's friends, boss, or parents. We discuss the distinct challenges of visual mementos by describing: (1) how each challenge responds to the purposes of visual mementos, (2) how visualizations can help address these challenges, and (3) how these challenges complement visualization systems for data analysis.

C1: Evoking FAmiliarity. Mementos can be a representation (e.g. photos, videos etc.) or a symbol (e.g. a souvenir, gift etc.) of an experience or person [46]. Visualizations have a strength in representing data and therefore lend themselves to the creation of representative mementos, though symbolic visual mementos can also be created. Representative mementos can be designed through a mapping that highlights patterns that correspond to the memories of the data owner. We define this as reflection of familiar patterns that can help to trigger personal memories and lead into episodes of reminiscing. In contrast to common analytical approaches that aim to uncover unknown patterns and support new insights, visual mementos can use this strength to reveal patterns that help the data owners recognize their own history in the visual mapping (as discussed in section 2.3).

C2: EXPRESSING SUBJECTIVITY. The constant re-interpretation of our memories is an important aspect of self-reflection [8, 12, 17]. Our autobiographical memory is not an objective storage of experiences, but rather a subjective interpretation of our past. People subjectively interpret the importance of a memory, the emotional aspects, and the personal narrative surrounding the memory. Visualizations can express subjectivity by giving data owners freedom to adjust the mapping, select and curate their data, adjust the appearance to their personal aesthetic taste, and attach personal narratives and symbolic meaning to the representation. This goal is specific to visual mementos, as visualizations usually aim to represent data more objectively.

C3: ObSCURing Sensitive Data. As visual mementos can be used for sharing personal memories with others, they should provide a means of hiding aspects that people may not want to reveal. This is especially true when data owners delegate collecting, selecting and mapping the data to a system. This challenge can be addressed on the data level or the representation level. Visual representations are useful tools for revealing information from raw data, but can also help to obscure fine-grained details e.g. through aggregation and abstraction. Interaction can give the data owner control over the amount of detail that is revealed in the visual memento. While more commonly visualizations aim to accurately represent data, in visual mementos it can be desirable to filter out aspects based on the intentions of the data owner.

\subsection{Personally Relevant Data Types}

To support the described purposes, visual mementos use data from different sources and logging systems. These tools give people varying amounts of freedom to specify when personally significant events are taking place. The aspect of personal significance and how it is captured in the data plays an important role in the creation of visual mementos, as emphasizing significant episodes can be a way to reflect personal interpretations of the past in visual mementos.

Data collected by a third party can be personally relevant if it has a relation with a person's history (e.g. data about a soccer game watched in the stadium). In this case, judgment of personal significance is applied when the data source is sought out and selected. More commonly, visual mementos are built on data that directly captures someone's personal history. Personal data can be collected selectively or continuously: Selectively captured data e.g. photos, status updates on social networks, or heart-rate logs of a run deliberately capture personally significant events. As people consciously decide what to capture and what to leave out, they apply subjective judgement of personal relevance in the collection process. Continuous Lifelogs can include data from always-on sensors (GPS, pedometers), lifelogging cameras (Autographer and SenseCam) or other software (listening histories, activity logs) running in the background. Since a computer logs the data, a measure of personal significance may be applied after the capture.

Since selectively collected data provides a subjective lens on life, most existing systems for reminiscing focus on this kind of data (photos etc.). Continuous lifelogging data is used less. However, we argue that both selectively captured as well as lifelogging data can be used to create visual mementos as our system, visits, exemplifies.

\section{Designing a Visual memento: An Example}

In this section, we present the design of a tool for creating visual mementos that exemplifies the purposes and challenges described in section 3. This example explores one instance of the many possible implementations of visual mementos. It allows us to demonstrate a proof of concept and reflect critically on its shortcomings and benefits. In the following we describe the design rationale and the system.

We decided on a GPS location histories because this type of data can encompass both, selectively as well as continuously captured data. To evoke familiarity we aimed to represent familiar patterns (C1) by using a visual mapping based on the structure of autobiographical memories 
from a previous research prototype [59]. Expressing subjectivity (C2) can be supported through various features, from simple customization to freeform expression. Rather than supporting a wider range of complex, though possibly expressive, adjustment features, we decided on simple customization to keep the system simple to use while offering some personal expression. Also, to give people the opportunity to obscure sensitive data when sharing $(\mathbf{C 3})$ we provided a feature that allowed them to adjust the level of privacy through visual aggregation.

The system allows people to load and visualize personal GPS location histories and share the created representation with others. We
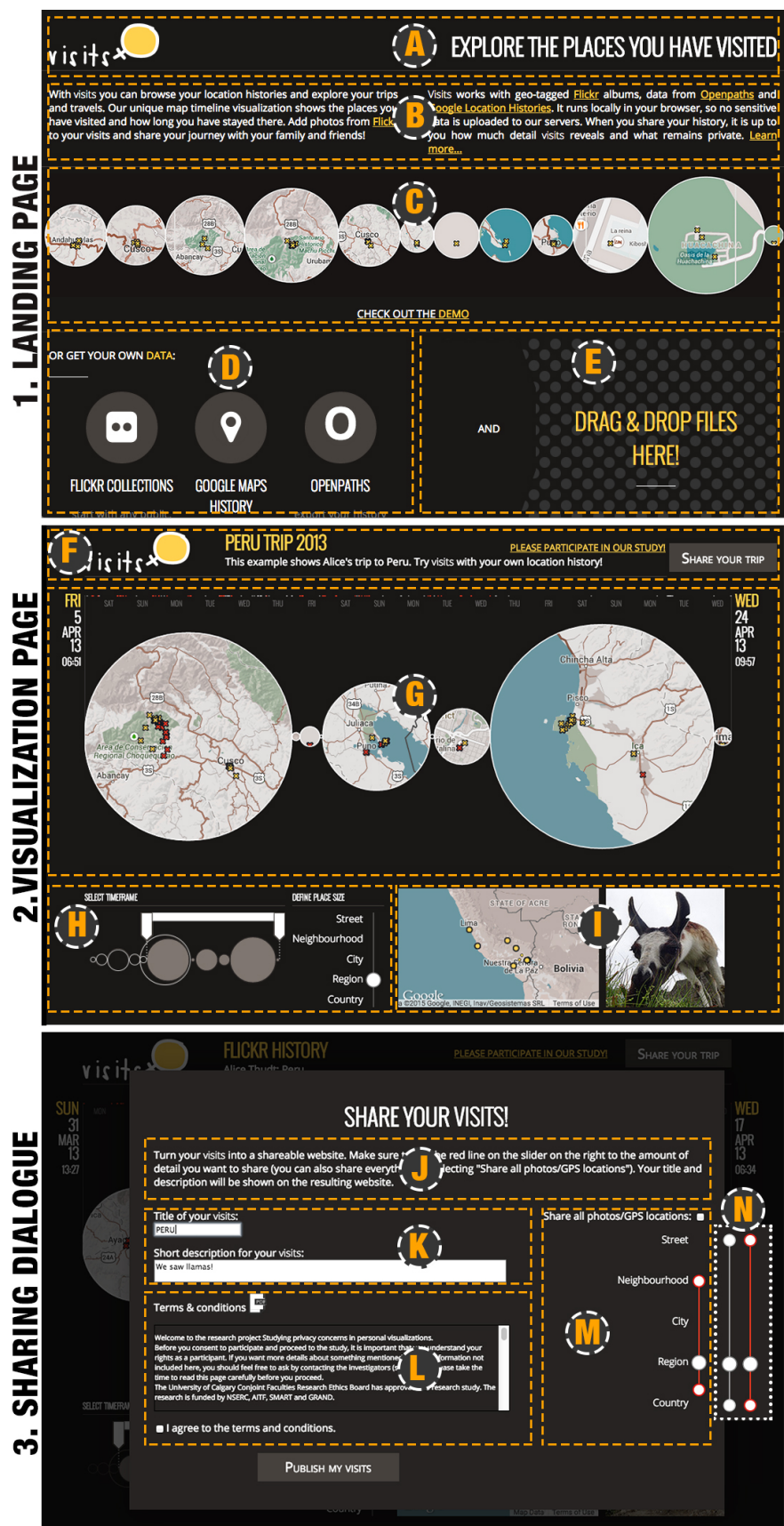

Fig. 1. The visits system: 1. Landing page with A) header, B) system description, C) teaser and demo link, D) third party data sources, E) data loading drop box. 2. Visualization with $F$ ) header with title, and share button, $G$ ) map-timeline, $\mathrm{H}$ ) controls for timeframe and place adjustment, I) overview map and photo box. 3. Share screen with: J) sharing description, K) textfields for title and description, L) sharing terms and conditions, M) privacy slider for limiting the shared details. $\mathrm{N}$ ) shows possible settings of the privacy slider for sharing all locations and locations from street to country level. envisioned the created mementos for reminiscing about experiences where personal movement is particularly memorable: trips and travels. Our goal was to build a tool that provides a means for people to reflect on their trips and share their experiences with their friends and loved ones. Although the system design focuses particularly on travel histories, we kept in mind other possible usage scenarios such as the exploration of everyday movement as well as long-term lifelogs.

Location histories can be logged by modern smart-phones, however, most people do not know how to access this data. Our online system supports people during the entire process of creating a visual memento: from extracting their data from third party services to visualizing and sharing it. Designing a fully functional web visualization system requires careful consideration of a simple, pleasurable, fluid and aesthetically appealing experience [36, 23]. For this reason we aimed to incorporate rewarding interaction as well as an attractive graphic design, and keep the features as simple as possible. To support different display sizes and devices, the layout of the page is responsive to the browser window size when loading the location history.

The system contains three main pages (see Figure 1): (1) the landing page to access and load personal data from third party services, (2) the visualization page where the data can be explored locally in the browser, and (3) the sharing page, that allows uploading data at the desired level of detail to the server to share created artifacts with others. We describe navigation and interaction with the system in a walkthrough example of Valentina, an imaginary site visitor.

\subsection{Landing Page and Data Loading}

Valentina arrives on the visits landing page (Figure 1-1). She reads the short description of the interface at the top of the page (Figure 1B). The text invites her to extract and load her data and assures her that no data will be uploaded unless she decides to share the visualization. Below, multiple options allow her to either try a demo of the interface (Figure 1-C) or load her own location history to create a visual memento. Valentina decides to load her own history. The system provides the following options: loading a geotagged photo collection from Flickr, or loading a kml or json file from Google or Openpaths (Figure 1-D). After downloading her two year Openpaths history, she drops the file onto a box on the landing page to load the data and visualize it (Figure 1-E). Once her history is loaded, Valentina explores her visual memento and realizes that she can add pictures from a Flickr collection, adjust various parameters to emphasize important moments (Section 4.2) and share her visualization (Section 4.3).

\subsection{Visualization Design}

In this section, we describe how we address the challenges of evoking familiarity (C1) and supporting the ability to express subjectivity in our visualization (C2). The visualization page (Figure 1-2) is composed of several components. The header displays a title and description of the visual memento. These elements can be adjusted when sharing. The main component is the map-timeline [59]. The visualization can be adjusted through controls positioned below the maptimeline. The two last components are an overview map and a photo box displayed on the bottom right.

\subsubsection{Visual mapping}

To address the challenge of mapping data to evoke familiarity (C1), we use a technique that uses a semantic clustering of GPS logs that reflects people's autobiographical memories (details in [59]). For the reader's convenience we briefly recap this visual mapping:

People often remember episodes that involve movement (e.g. trips and travels) as a sequence of stays at different places [61, 10]. Places can be defined as areas of variable size (e.g. a building, city or country) and stays are time periods spent within one "place" (e.g. "my 2-week stay in Europe" or "my 8-hour stay at the lab"). For a mapping that supports reminiscing we chose a representation that shows movement data as a sequence of stays, similar to our autobiographical memories.

The map-timeline visualization was deliberately designed to put spatial and temporal aspects on equal footing by showing: (1) the chronological order of stays, (2) repeated visits, (3) duration of stays, 
and (4) movement during a stay. Map-timelines organize circular map segments along a linear temporal axis. Each circular map segment represents a stay and its diameter represents the duration of this stay from the first logged location inside the place to the last. The zoom level is chosen to include all logged locations within the place. Locations are represented by cross markers for individual logs to show movement during one stay. This results in a visualization that visually emphasizes places visited for a longer time (which potentially have greater personal significance) while downplaying locations passed through in transit. The map-timeline is annotated with a subtle temporal axis at the top, showing the distribution of logs over time, and an overview map at the bottom, showing all visited places (Figure 1-2-G).

\subsubsection{Customization and Interaction}

To support the expression of subjectivity (C2), we aimed to support simple customization for emphasizing episodes of personal significance. The map-timeline visualization highlights longer stays, assuming that those are personally more significant. We enhanced this inherent emphasis with features that allow customization of the mapping by changing the displayed timeframe and defining what is considered as a place. We also support adding photos to a created memento. In this section we present these features and describe how Valentina interacts with the visualization and reflects on the represented time period.

Selecting a Timeframe: During her exploration, Valentina decides to hone in on a 2-week trip to Italy to reminisce. A widget at the bottom-left (Figure 1-H) allows her to limit the displayed timeframe by dragging two handles. She notices that she can also drag the bar between the handles to pan the view. The timeframe widget displays a miniature version of a simplified map-timeline showing her two year history. This widget supports accurate selection of a specific timeframe. When Valentina selects the 2 weeks of her trip in the widget, the map-timeline displays a smooth animation transitioning the view to this timeframe. This transition helps to create fluid interactions that support casual exploration [23]. The circles representing her Italy trip are now shown in the central timeline and the widget highlights them in grey while the rest of her two year history is shown as circle outlines. To further explore a weekend she spent in Rome, she clicks on the corresponding circle and the visualization zooms in on this stay.

Defining Place Size: As explained earlier, the colloquial concept of "place" can be interpreted in different ways, e.g. Valentina remembers her stay in Rome as a sequence of visits of historic sites but her whole trip to Italy as a sequence of stays in different cities. A slider next to the timeframe widget (Figure 1-H) allows her to define what she considers a "place" to bring the representation closer to her subjective and personal interpretation and allows her to explore her trip on different levels. She drags the place slider to "street" to show the different sights she visited in Rome. As she drags the slider, the central map-timeline displays a simplified preview of the new clustering as grey filled circles, while the previous configuration is displayed as white outlines. We designed this interaction to allow Valentina to judge in advance how places will separate into smaller places. When she releases the slider, the corresponding map segments are loaded.

Loading Pictures: The system allows location histories to be enhanced with geotagged photos from Flickr. This adds an additional subjective aspect in form of selectively collected data. Valentina notices the photo box in the bottom right corner labeled "Add Flickr Photos" (see Figure 1-I) and decides to add a Flickr collection of her Rome weekend. She clicks the box and a dialog appears where she provides her Flickr username and selects her public Rome collection. After her images are loaded, the associated geotags are integrated as red location markers in the map-timeline and the photos appear in the photo box. She moves the mouse horizontally over the photo box to browse her photos in chronological order and stops on a picture of the Colosseum. She clicks it and the picture is enlarged in a popup overlay.

Exploration through Hover: When Valentina hovers over the central map-timeline, additional information about her individual stays are displayed. She stops on a circle showing the Piazza Venezia: the start and end time of her stay are highlighted on the time axis and the name of the place is displayed above the time axis. A curved line connects the map-circle to the corresponding location marker in the overview map. When Valentina hovers over a cross location marker within a map-circle it is enlarged and a dotted curved line connects the marker to the corresponding time point on the time axis. When she hovers horizontally over the map-timeline she can explore location markers in chronological order. This supports both the exploration of temporal and location aspects without having to change the mode of interaction.

\subsection{Sharing with Adjustable Privacy}

Location histories can contain sensitive details about an individual's personal life. The privacy of this data is an important design consideration (C3), therefore, we support local exploration as well as adjusting the level of revealed detail when sharing. We make visitors aware of these features by explaining them on the landing page (Figure 1-B).

Until this point Valentina has explored her data locally in her browser. No sensitive data is uploaded to a server. As Valentina is satisfied with the display of her weekend trip, she decides to share the created memento with her friends and clicks the "Share your trip" button (Figure 1-F on the right). This brings up the sharing dialog (Figure 1-3) where she can provide a title and description of her memento and agree to upload her data to our server.

Visits further provides a feature that allows her to adjust how much data details are revealed when the visual memento is shared. Reading the description (Figure 1-J) Valentina notices that she can adjust the level of detail that is uploaded and revealed in her shared memento by using a slider with two handles (Figure 1-M). She limits the aggregation levels on which her friends can explore her shared memento by setting the bottom handle to neighbourhood and the top handle to region. This results in a visual memento that her friends can explore on neighbourhood, city and region level. In the background, the system creates a new location history that only contains the defined level of information. In this way, Valentina does not have to share her entire history with a third party. The map-timeline does not include markers of individual logs, unless Valentina decided to check the option "Share all photos/GPS locations". To finalize her sharing Valentina clicks the "publish your visits" button and the data is uploaded in the specified levels of granularity. A popup window displays the unique URL of the visual memento alongside social media buttons. Valentina decides to directly post the created memento on Facebook. She also copies the URL for accessing her memento later and sending it by email to her mother who is not on Facebook.

\section{Case Studies of Visual Mementos}

To provide a general understanding of how people used the system in a realistic online deployment, we present case study examples of visual mementos that people shared online. The 18 shared visual mementos show a great variety of use cases and illustrate how people appropriated the system for diverse purposes, some of which we had not envisioned. In the following, we illustrate this variety with six quite delightful and surprising examples (overview in Figure 2).

Mementos of Short Term Trips. The memento with the title "Toronto, Burlington, Montreal, and Ottawa -A quick spring jaunt through eastern Canada with a stopover in Vermont." (Figure 2-1) shows a two week trip that comes very close to our envisioned use case for visits. The two main stays of this trip - Toronto and Montreal - are clearly visible and show the prominence of these places during the depicted trip. Places that were passed through in transit are shown as less prominent circles in between. Similar examples show a three week trip to Egypt and Jordan, a two day trip to Cambridge and a five day road trip in southern US entitled "Pre Christmas Ingress RoadTrip Fun". The short timeframes that exclude long stays at home suggest that these mementos deliberately extract personally significant events.

Memento of an Activity. The memento shown in Figure 2-2, depicts an even shorter episode of personal significance: a walk in the park. The memento with the title "My walk in the English Garden On 27/4/2014" (translated from German) shows the shortest activity shared online. The creator chose to include all logged locations in his representation, to show all details of the walk. This example shows that limiting the shared timeframe to episodes that do not contain sen- 

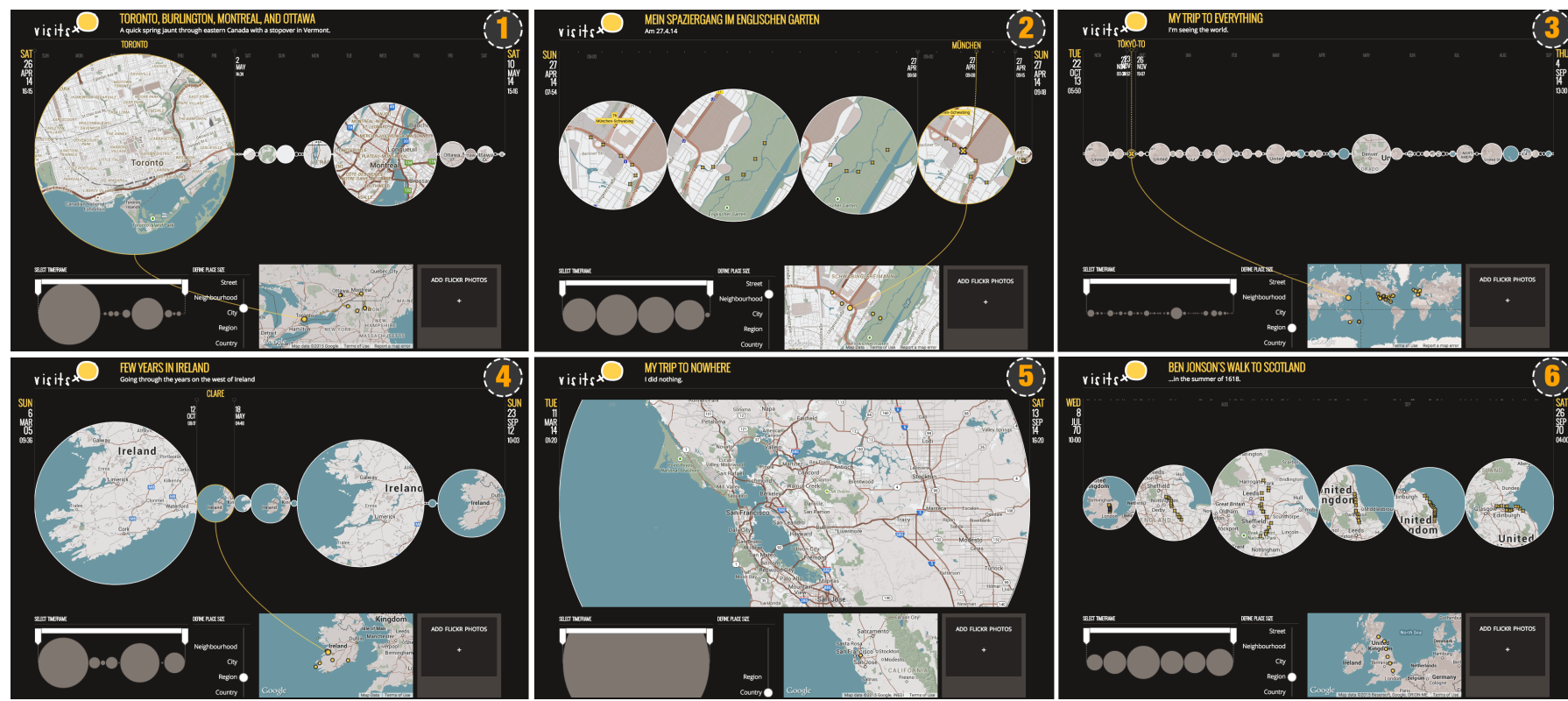

Fig. 2. Case studies of mementos of 1) a short term trip: "Toronto, Burlington, Montreal, and Ottawa - A quick spring jaunt through eastern Canada with a stopover in Vermont.", 2) a walk in the park: "My walk in the English Garden - On 27/4/2014", 3) multiple trips: "My trip to everything - I'm seeing the world", 4) everyday life: "Few years in Ireland - Going through the years on the west of Ireland", 5) boredom: "My trip to Nowhere - I did nothing", and 6) a historic event: "Ben Jonson's Walk to Scotland - in the summer of 1618".

sitive details can be another way of maintaining privacy without having to visually obscure locations.

Memento of Everyday Life. The memento that covers the longest time period is the example shown in Figure 2-3 entitled "Few years in Ireland - Going through the years on the west of Ireland". As the title as well as the duration suggests, this memento does not focus on a specific experience, but rather depicts a holistic view of the creator's past. As our design did not specifically focus on such use cases, the visual representation is not as effective: it shows multiple very similar map segments in the map-timeline. This drawback points to opportunities for future improvement, such as using specific algorithmic mappings for different types of histories.

Memento of Multiple Trips. The trip with the title "My trip to everything - I'm seeing the world" (Figure 2-4), shows the greatest geographical variety including stays in North America, Europe, Asia, Australia and New Zealand. The title hints at an intention to show how well-traveled the creator of this memento is and how many places s/he has seen during one year. While the overview map shows an impressive number of visited locations, the map-timeline is less effective as it shows the significant trips as small circles between multiple bigger circles representing returns home to North America - a problem that was also raised by participants in our questionnaires (Section 7.4).

Memento of "Boredom". An unexpected instance is the memento shown in Figure 2-5. The creator gave this 6 month history the title "My trip to Nowhere - I did nothing". The only map-segment in the map-timeline shows the San Francisco Bay. This example is a counterpoint to the previous memento of world travel. While the first implies an exciting life with many memorable experiences, in the latter, the subtitle "I did nothing" communicates emptiness or boredom. The creator further emphasized this message by using the customization and privacy features: Setting the place size to country and the maximal level of explorable detail to region, hides all movement and creates little visual variation in the memento, which can suggest monotony.

Historic Memento. Another surprising memento, shows historic movement data: "Ben Jonson's Walk to Scotland - in the summer of 1618" (Figure 2-6). This instance is the only example of a representation that does not show personal movement but rather represents data that has a different relevance for the creator. This extends possible use cases for visits beyond personal reminiscing to showing other datasets of personal interest. The memento of Ben Jonson's walk is the only example of an artifact created in honour of a historic person.

\section{Studying Visual Mementos}

We used visits to study people's reactions to visual mementos. We start by explaining the choice and application of our study methodology: a technological probe. We then outline our data collection. In the next sections, this is followed by our results and a discussion of the challenges in light of these results.

\subsection{Choosing a Methodology}

Studying personal visual mementos is challenging for several reasons. First, the recruitment of participants who own the necessary personal dataset, know how to access it and are willing to provide it for study purposes, can be difficult. Second, traditional measures of efficiency and accuracy might not be the most meaningful way to study visual mementos, as their effectiveness strongly depends on people's experience. This experience is highly dependent on the usage context and therefore hard to investigate in a lab setting. In their survey of personal visualization systems, Huang et al. found that many evaluations of such systems still rely on controlled studies with predetermined tasks and sample data [31]. We argue that studies of visual mementos have to go beyond this, as their main purposes - reminiscing, self-reflection and sharing of personal experiences - are strongly dependent on subjective perceptions and experiences.

In this study our objective is to find out if our example of a visual memento can be used for these purposes. Lam et al. [34] present and explain different scenarios for performing an empirical evaluation of information visualizations. However they do not provide specific guidelines for studying our specific new application domain. For this reason we choose a method used by researchers who are studying digital mementos $[33,68]$ and is well-adopted in the Human Computer Interaction community: a technology probe [9]. A technology probe is often a particular artifact or group of artifacts distributed as a means of collecting qualitative information to understand people's reactions, usage and needs. It can also be used to demonstrate a proof of concept through the introduction of new technology in real-life scenarios and inspire future design. Probes are useful tools to collect emotional or subjective feedback, which was the intention of our study. Our probe is the fully functional web system described in section 4 . This system is used to collect contextual feedback from participants describing their subjective experience. The main questions we investigate are:

- Did our system support the purpose of reminiscing?

- Is our system used to share visual mementos?

- Do people recognize familiar patterns and experience the visual- 
ization as reflecting their own memories?

- Do people find the customization features sufficient for representing a subjective interpretation of their past?

- How do people feel about privacy and do they obscure details of their data when sharing?

\subsection{Data collection}

To respect our participants' trust and to study the challenge of adjustable privacy we could not actively record any information that reveals personal details without participant consent. We considered that showing a disclaimer to get participant consent upfront would impact their experience and the experimental condition as this is already a form of sharing. For this reason, we carefully avoid recording any possible sensitive information or behaviour that could reveal any personal information. We explicitly state this on the homepage to inform visitors that until they share, no information is uploaded to the server. This limited our observation capacities but also allowed us to study an ongoing system in an unobtrusive way.

We collected data during a period of seven months. Participants were recruited by email, postings on social media sites and in online communities with an interest in visualization and personal data collection. We distributed postcards advertising our system and study. The application was covered by the specialist blog community and some press websites. This further helped us to attract participants.

We designed a mostly freeform online questionnaire to assess people's reactions to the system and to investigate our research questions. It included questions about: the type of location history people explored in the system (e.g. long term or travel histories, etc.); a Likert scale of how well the visualization reflects their memories; a selection for their sharing and privacy preferences; and freeform questions about their satisfaction with the system's features, sharing and personalization. We also gathered usage logs with Google Analytics ${ }^{6}$. In addition, we analyzed 18 visual mementos that people created and shared.

\section{RESULtS}

In this section, we describe the results of our analysis of questionnaires and shared mementos. We first describe the general use and reception of the system. We then analyze how the system supports the purposes of reminiscing (P1) and sharing (P2) and how well it addresses the challenges of mapping for familiarity (C1), expressing subjectivity (C2) and maintaining privacy by obscuring details for sharing (C3).

\subsection{Usage Details \& General Feedback}

According to Google Analytics, during our study, the system received 8168 unique visitors. Of this population $46 \%$ were women and $54 \%$ men, with $60 \%$ of people aged between 18 to 34 years, and $18.6 \%$ of this population returned after a previous visit. Visitors spent an average of 2.54 minutes on the site. 509 visitors spent more than a minute on the website and 115 stayed more than 10 minutes. We consider visitors as study participants if they filled out a questionnaire (25 participants) or shared a memento (18 participants) or did both (1 participant). 12 participants filled out the questionnaire after trying the demo, 12 answered after exploring their own data and 1 replied after exploring and sharing a memento. This participant is also counted in the 18 sharers. In total we count 42 participants.

The data that participants explored in the system was mostly collected during everyday life ( 8 out of 12). Only 2 people explored data that was collected solely during travel. Most participants stated that their location histories covered several trips to different locations.

The engagement with the system as well as the reply rate to our online questionnaire was lower than expected. However, people who loaded their own data provided enthusiastic comments about the system: "It's an awesome visual representation of my travels [...] Thanks for creating this and pushing the boundaries of personal data exploration!" [P6], "I love the app! Such a cool combination of place and time in one view." [P4]. Participants further described the application as "Fun to explore" [P9] and found that the system "makes it easy to

${ }^{6}$ http://www.google.com/analytics/ see where you've been" [P7]. A lot of comments concerned the attractive aesthetics of the visualization, describing visits as "beautiful" [P9], or "very elegant" [P22] and lauding the unusual mapping [P9].

\subsection{Reminiscing $(\mathbf{P} 1)$}

Participants stated that the mementos created in visits triggered memories and explained how it reflected their personal view of the past: "Visits conveys a kind of view on geo memory I think wouldn't get otherwise." [P10]. Some participants mentioned that the system helped them assign meaning to previously mundane aspects of their day to day life, e.g.: "It was interesting to see how I stayed in my 'normal' location (work/home) quite a lot, but the tool also allowed me to explore even more transient locations. Although they were represented rather small in the timeline, by hovering over the circles, I was able to quickly make sense of these more transient places which triggered memories. In a way, those more transient places are more interesting during everyday life because they show trips that break out of the daily routine." [P2]. This seemingly positive comment on interaction and visualization concept also hints on potential problems with the visual mapping that make subjectively more interesting episodes (e.g. travel) appear small. Other participants stated that the system provided them with insights about their lives: "I find the patterns that come out of the tracking to be pretty compelling." [P22] "It allows interesting observations about travel patterns and life trends". [P6]

\subsection{Sharing of Experiences (P2)}

During our seven month study period, few people used the sharing feature. Overall, only 18 people of the 509 who stayed on the site for more than one minute shared their memento. Among the participants who completed the questionnaire, only one person had already shared their memento. Six of the 11 who filled out the questionnaire after exploring their own data stated that they plan to share in the future. This suggests that online sharing was not popular, however, we were able to collect some interesting details about what people shared.

Shared Mementos: People shared mementos of location histories covering a variety of countries in Europe, Canada, the US, Asia, and Oceania. Shared episodes ranged from short activities such as walks in the park, over travels to long term depictions of everyday life. The shortest history shared as a memento covered a time period of 1.4 hours, while the longest spanned seven and a half years. On average, shared histories were 9.14 months long. When sharing their history, 15 out of 18 participants provided a title for their memento and thirteen changed the subtitle. Since only one person who shared their memento also filled in a questionnaire, we have no information on how the created visual mementos were used and who people shared them with.

Souvenirs for Storytelling and Joint Reminiscing: While the sharing feature was rarely used, participants' comments imply that visits could be used as an artifact for joint experiences. Some participants stated in their comments that they wanted to display the visual memento in their home to represent memories and encourage discussions e.g: "It is a beautiful piece in itself. I would like to have it for myself as a piece of personal art. Hanging it somewhere may trigger some conversations with friends about certain trips." [P2] The same participants did not use the sharing feature included in the system. This unexpected result suggests that our feature did not match people's expectation and existing practices with mementos, but it reveals that visual mementos could be effective in supporting a social function: as an aesthetic artifact for joint reminiscing or a trigger to "to tell a story" [P10]. Comments further suggest a desire to use the system for creating artifacts collaboratively with others, a feature we did not integrate: "Say I go on a trip with 12 people, we might all want to get together and create an aggregate visualization of our trip" [P1].

\subsection{Evoking Familiarity (C1)}

We have identified the capability to evoke familiarity as a main challenge of visual mementos, as it supports reminiscing. A visualization can address this challenge by providing a representation that reflects people's personal memories of the displayed timeframe. We asked participants who loaded their own data in visits how well the visualization 
reflects the stays they remember. We used a Likert scale to collect their answers with possible responses ranging from 1 (not at all reflected) to 5 (very well reflected) with an average of 4.44 . This high score suggests that people who explored their own data found their memories well-depicted by the visual representation. Participants' additional comments emphasize this further, for instance: "It spurs memories of particular events. [...] I loved looking back and recognizing the time that I was actually in one place for an extended period" [P4].

We found that the type of personal location history influenced how well participants' memories were reflected. Participants explored both continuous lifelogs and selective short-term travel histories and combinations containing both everyday movement and travel in visits. The design of visits mostly focused on travel memories, therefore, some participants who explored everyday movement or combinations of travel and lifelogs found the visual representation less appropriate for emphasizing moments of personal significance. For instance, one participant noticed the fact that longer stays at their home are represented quite prominently in the interface compared to more personally significant episodes of travel: "It is focusing very much on the in between places. The things I drove through and not the important locations where I most probably spent longer. My trip to Serbia [...] is really not reflected well. Other things like my travels between Berlin and my home town are highly visible. The outliers are the important travels for me." [P4]. This suggests that giving greater visual weight to longer stays, works well for travels but is less effective for everyday movement. When reflecting on longer continuous lifelogs, our mapping tends to highlight the mundane rather than the extraordinary trips.

Reflecting memories could also be challenging when the data is not accurate due to technical problems that lead to logging errors or holes in the data. While this problem cannot be entirely avoided in representations of automatically logged data, it can also impede the reflection of memories: "The accuracy of the underlying data is a bit off sometimes - particularly when I'm in Auckland. I think it's because I spend more time in a car there, and my phone's GPS doesn't work so well." [P7] "I had no Internet access in Stockholm therefore the logs were less than useful.". [P14].

\subsection{Expressing Subjectivity (C2)}

Allowing customization of the mapping to bring it closer to the subjective interpretation of one's past is an important feature in our system. Participants appreciated the possibility of adjusting the place size: "I loved [...] being able to change the level of aggregation." [P6]. One participant mentioned that this feature helped to get different views of their past: "I really liked how the location granularity can be adjusted because different time frames can be viewed from different perspectives." [P2]. Our analysis of shared mementos also shows that people made use of this customization feature: Seven out of 18 changed the preferred place size to neighbourhood, three adjusted it to city, and two to country. For the six people who shared the memento on region level (the default setting) we could not determine if they simply did not use the customization feature or if they considered it the most appropriate setting for their history. However, the fact that 12 out of 18 sharers adjusted the place size suggests that this feature was useful. Participants further appreciated our feature for selecting specific timeframes from a longer-term history. Comments suggest that it supported participants in exploring episodes that they wanted to remember: "For instance, I was looking at my entire location history (more than a year) but then selected certain time periods where I knew I was traveling. It would be useful to have some more time labels in the 'select timeframe' widget. But without it, I was able to allocate certain times by looking at the circle sizes and order in the big timeline." [P2].

While people generally appreciated our simple customization, some participants also experienced it as limiting. They provided further suggestions for personalization including control over the graphic representation and integration of additional data sources. This suggests that expressing subjectivity can go beyond simple customization in visual mementos. Further, participants raised concerns about the inaccuracy of automated logs and the notion of forgetting as an important part of remembering. While the customization feature allows adjusting the granularity of aggregation and selecting a timeframe, it does not allow emphasizing subjective importance and removing undesired parts.

Memory Accuracy and Forgetting: The human memory is a selective capture of personally significant experiences, rather than an exact recording of events. Defining what is worth remembering and what can be forgotten is important to construct a personal interpretation of the past. When designing the system we did not consider the aspect of forgetting, but we received some unexpected comments that illustrate how depictions of personal data can trigger forgotten memories: "visits provided some information I forgot, so it doesn't reflect my memory. [...] I mean [it shows] some information about places I have been to, and time I spent there during the last month, that I totally forgot." [P10]. This can be desirable in the context of reminiscing, and lead to moments of self-reflection, or complicate the experience of the visualization as a subjective representation of one's past.

\subsection{Obscuring Sensitive Data (C3)}

The last challenge for visual mementos is to maintain privacy of data owners by letting them adjust the level of represented details. We first present the analysis of people's privacy preferences and then describe how they used the feature for adjustable privacy implemented in visits.

How much detail of your location history would you share with whom?

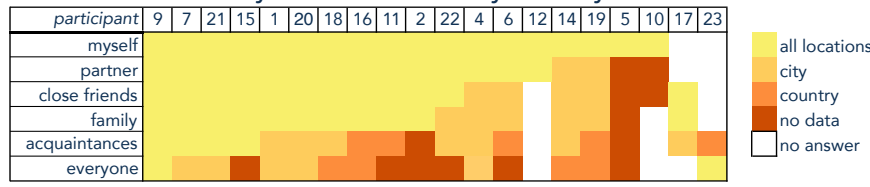

Fig. 3. People are willing to share location histories at varying levels of detail with different people. In this figure, only participants who answered this question are displayed.

We asked all participants "How much detail of your location history would you share with whom?". As answer options we provided different levels of detail (all locations, locations at city level, country level and no location data). For each level of detail we let people pick with whom they would share this information, ranging from intimate (self and partner), friends and family, to acquaintances and public (sharing with everybody). The results suggest that sharing an artifact based on movement data is a delicate matter and privacy concerns seem to vary between participants (Figure 3). For instance, we found that 4 people were not willing to share all details with their partners. In this group, two people did not want to share any data with anyone. On the other hand, one person stated that they would share all data with the public, but only on country level with acquaintances. This could be due to perceived anonymity when sharing data with unknown people on the web. 14 participants were willing to share all locations with their partners, 11 with close friends and 10 with their family.

We further analyzed the 18 mementos that participants shared with regard to the chosen privacy settings: Only four people chose to include all location details in the representation. Nine people made their shared memento explorable on street level but without including the details of individual logs. Four people limited the level of explorable detail further to neighbourhood level, and the most restrictive memento was only explorable on region level. This shows that people who chose to share their memento cared about keeping certain aspects of their data private and that our system supported them in doing so.

\section{Discussion AND FUtURE WORK}

Technology probes do not provide generalizable observations. Our methodology did not include logging of interactions which makes it difficult to describe people's behaviour. The assessment of reminiscing is solely based on self-reported comments which limits the precision of the evaluation. However, the technology probe shed light on people's needs and desires in a real-world setting, enabled the testing of an application in the field, and can inspire future designs. In this section we describe limitations and benefits based on participant reactions to our probe, and derive dimensions for future research in the application area of visual mementos. 
Visual Mappings for Different Memories. In our example system for creating visual mementos, we explored one visual mapping of location histories for reflecting travel memories. While the general engagement with the system was low, the people who explored their own data created visual mementos of a variety of different memories ranging from everyday movement to short activities and longterm life patterns. Comments suggest that the used visual mapping did not work equally well for all types of histories people explored, which could potentially explain the low uptake. New mappings might be more suitable and can be explored further in future research. Additionally, people collect data about other aspects of their lives, e.g. their social interactions and relationships or their hobbies. It remains an open challenge to find adequate mappings for reflecting people's memories of other types of personal experiences and based on different datasets. Our initial exploration opens up a new space for further investigation of how visual mappings can be used to reflect aspects of people's lives. Also, the creation of symbolic visual mementos needs further investigation.

Freedom for Personalization. The customization features of the presented interface are limited compared to the freedom provided in the creation of physical mementos. Despite these limitations, our study unveils how people appropriate the provided features creatively to show a subjective interpretation of their past. Additionally, their comments suggest merit in providing more freedom to make a visual mementos truly personal. Such features could support changing the visual appearance to personal preferences of aesthetics, annotation and personal storytelling, emphasizing subjective importance or removing undesired aspects and adjusting or even defining the visual mapping. Personal visual mementos created by professional visualization designers [25, 39] exemplify what such truly personal and subjective interpretations of the past could look like. Designing more expressive features that support the subjective adaption and creation of visual representations, and are accessible to a broader audience, is a research challenge of visual mementos that needs further investigation.

Supporting Joint Reminiscing. The presented system only considers individual creation of visual mementos for personal reminiscing or sharing with a remote audience. This is a limitation of the system that participants specifically mentioned. Their feedback suggests the consideration of a wider spectrum of use cases for visual mementos. Future systems could allow people to merge multiple histories or draw from shared data sources, to create a collective visual mementos. Systems could further support joint reminiscing and co-located storytelling about individual or shared experiences similar to the common practices with physical mementos and souvenirs. From these suggestions, we identified the support of joint reminiscing and collaborative creation of visual mementos as promising avenues for future research.

Visual Mementos Beyond the Digital. In Visits the created visual mementos can be made available on the web. Participants' statements suggest that they are longing for a representation that they can better integrate into their home where it can trigger serendipitous reencounters and stimulate communication with friends, which our system did not incorporate. Future research on visual mementos could explore possibilities to free visual mementos from the computer screen. This could, for instance, be achieved by supporting high-resolution printing, or creating tangible mementos of personal data (as e.g. in [56]) that can be carried around or displayed in the home.

Studying Visual Mementos. Visual mementos have inherent hurdles that complicate their adoption and assessment: (1) people might not collect the required data or know how to access it, (2) loading personal data to explore a web visualization requires more initial commitment than when encountering other casual visualizations on the web, (3) since personal data contains sensitive information, privacy concerns can further limit the number of people willing to provide this data. Our evaluation is a rare attempt to study personal visualization in the field, which makes it hard to compare the engagement and impact to other instances. Despite the low number of people who engaged with the sharing features, people who overcame the entry hurdles provided positive comments. This suggests the merit of the concept of visual mementos as well as our example. Future studies of personal mementos can mitigate these evaluation challenges by combining studies in the field and in the lab. Another possibility to overcome the discussed entry hurdles and achieve higher engagement would be to integrate the visual memento system with the data source (as e.g. in [5]).

\section{Conclusion}

We have introduced visual mementos as a new visualization application area. From an extensive literature review of related research in human-computer interaction and personal visualization, we identified the following specific challenges for visual mementos: (C1) evoking familiarity, (C2) expressing subjectivity, and (C3) addressing people's privacy concerns by obscuring sensitive data.

We further contribute an investigation that shows the potential of visual mementos and reveals promising avenues for future research. To elicit people's contextual feedback and subjective experience we employed a technology probe, which enabled people to build visual mementos based on their personal movement data. Our analysis of their shared thoughts, desires and use of the system shows the benefits of visual mementos. This investigation can be considered a proof of concept for the application domain of visual mementos. Our findings help to consolidate the definition of this application area and to extract limitations and dimensions for future investigations in this space.

On a global level, people are collecting gigabytes of personal data about their lives, which leads to great potential interest in visual mementos. Using visualization to browse this information for personal as well as shared reminiscing can be a step towards creating meaning with personal data and reintroducing richness and emotional resonance into our experiences with digital data collections.

\section{ACKNOWLEDGMENTS}

We wish to thank our participants and reviewers as well as Charles Perin and Lindsay MacDonald for their invaluable feedback. We also thank our funding agencies NSERC, SurfNet, AITF, Grand, Smart Technologies, that made this research possible.

\section{REFERENCES}

[1] G. Andrienko, N. Andrienko, and S. Wrobel. Visual analytics tools for analysis of movement data. ACM SIGKDD Explorations, 9(2):38-46, 2007.

[2] R. Banks and A. Sellen. Shoebox: mixing storage and display of digital images in the home. Proceedings of TEI, pages 35-40, 2009.

[3] G. Barata, H. Nicolau, and D. GonçAlves. Appinsight: what have i been doing? Proceedings of AVI, pages 465-472, 2012.

[4] J. S. Bauer, S. Consolvo, B. Greenstein, J. Schooler, E. Wu, N. F. Watson, and J. a. Kientz. Shuteye: encouraging awareness of healthy sleep recommendations with a mobile, peripheral display. Proceedings of $\mathrm{CHI}$, pages 1401-1410, 2012.

[5] D. Baur, A. Butz, and S. Carpendale. Arcs.fm - a backdrop visualization for music talk. EuroVis Short Papers, 2012.

[6] D. Baur, F. Seiffert, M. Sedlmair, and S. Boring. The streams of our lives: visualizing listening histories in context. TVCG, 16(6):1119-1128, 2010.

[7] C. G. Bell, J. Gemmell, and J. Haag. Total recall: how the e-memory revolution will change everything. Dutton New York, 2009.

[8] S. Bluck and L. J. Levine. Reminiscence as autobiographical memory: a catalyst for reminiscence theory development. Ageing and Society, 18:185-208, 31998.

[9] K. Boehner, J. Vertesi, P. Sengers, and P. Dourish. How hci interprets the probes. In Proceedings of CHI, pages 1077-1086, 2007.

[10] N. Brown and D. Schopflocher. Event clusters: an organization of personal events in autobiographical memory. Psychological Science, 9(6):470-475, 1998.

[11] J. Bruner. The narrative construction of reality. Critical Inquiry, 18(1):pp. 1-21, 1991.

[12] F. Bryant, C. Smart, and S. King. Using the past to enhance the present: boosting happiness through positive reminiscence. Journal of Happiness Studies, 6(3):227-260, 2005.

[13] M.-C. Chiu, S.-P. Chang, Y.-C. Chang, H.-H. Chu, C. C.-H. Chen, F.-H. Hsiao, and J.-C. Ko. Playful bottle : a mobile social persuasion system to motivate healthy water intake. Proceedings of UbiComp, pages 184-194, 2009.

[14] E. K. Choe, N. B. Lee, B. Lee, W. Pratt, and J. Kientz. Understanding quantified-selfers' practices in collecting and exploring personal data. In Proceedings of CHI, pages 1143-1152, 2014. 
[15] G. Cohen and M. A. Conway. Memory in the real world. Psychology Press, 2007

[16] S. Consolvo, D. W. Mcdonald, T. Toscos, M. Y. Chen, J. Froehlich, B. Harrison, P. Klasnja, A. Lamarca, L. Legrand, R. Libby, I. Smith, and J. A. Landay. Activity sensing in the wild : a field trial of ubifit garden. In Proceedings of CHI, pages 1797-1806, 2008.

[17] M. A. Conway and C. W. Pleydell-Pearce. The construction of autobiographical memories in the self-memory system. Psychological Review, 107(2):261-288, 2000.

[18] K. A. Cook and J. J. Thomas. Illuminating the Path: The Research and Development Agenda for Visual Analytics. IEEE Computer Society, 2005.

[19] M. Csikszentmihalyi and E. Halton. The meaning of things: domestic symbols and the self. Cambridge University Press, 1981.

[20] M. Czerwinski, D. W. GagE, J. Gemmel, C. C. Marshall, M. A. PérezQuinonesis, M. M. Skeels, and T. Catarci. Digital memories in an era of ubiquitous computing and abundant storage. Communications of the ACM, 49(1):44-50, 2006.

[21] J. Donath, A. Dragulescu, A. Zinman, F. ViéGas, and R. Xiong. Data portraits. Leonardo, 43:375-383, 2010.

[22] P. Eakin. How our lives become stories: making selves. Cornell University Press, 1999.

[23] N. Elmqvist, A. V. Moere, H.-C. Jetter, D. Cernea, H. Reiterer, and T. Jankun-Kelly. Fluid interaction for information visualization. Proceedings of IV, 10(4):327-340, 102011.

[24] D. Epstein, F. Cordeiro, E. Bales, J. Fogarty, and S. Munson. Taming data complexity in lifelogs: exploring visual cuts of personal informatics data. In Proceedings of DIS, pages 667-676. ACM, 2014.

[25] N. Felton. Feltron annual reports, 2013 (accessed March 30, 2015). http: //feltron.com/.

[26] D. Frohlich, A. Kuchinsky, C. Pering, A. Don, and S. Ariss. Requirements for photoware. Proceedings of CSCW, page 166, 2002.

[27] J. Gemmell, A. Aris, and R. Lueder. Telling stories with mylifebits. In Proceedings of ICME, pages 1536-1539. IEEE, 2005.

[28] R. Gulotta, W. Odom, and J. Forlizzi. Legacy in the age of the internet : Reflections on how interactive systems shape how we are remembered. In Proceedings of DIS, pages 975-984, 2014

[29] S. Hangal, M. S. Lam, and J. Heer. Muse: reviving memories using email archives. Proceedings of the symposium on User interface software and technology, pages 75-84, 2011.

[30] O. Hilliges and D. Kirk. Getting sidetracked: display design and occasioning photo-talk with the photohelix. Methodology, pages 1733-1736, 2009.

[31] D. Huang, M. Tory, B. A. Aseniero, L. Bartram, S. Bateman, S. Carpendale, A. Tang, and R. Woodbury. Personal visualization and personal visual analytics. TVCG, 21(3):420-433, 2015.

[32] V. Kalnikaite and S. Whittaker. A saunter down memory lane: digital reflection on personal mementos. International Journal of Human Computer Studies, 69:298-310, 2011.

[33] D. S. Kirk, S. Izadi, A. Sellen, S. Taylor, R. Banks, and O. Hilliges. Opening up the family archive. Proceedings of CSCW, pages 261-270, 2010.

[34] H. Lam, E. Bertini, P. Isenberg, C. Plaisant, and S. Carpendale. Empirical studies in information visualization: seven scenarios. TVCG, 18(9):1520 1536, 2012.

[35] J. E. Larsen, A. Cuttone, and S. L. Jørgensen. Qs spiral: visualizing periodic quantified self data. In CHI 2013 Workshop on Personal Informatics in the Wild: Hacking Habits for Health \& Happiness, 2013.

[36] A. Lau and A. Vande Moere. Towards a model of information aesthetics in information visualization. In Proccedings of $I V$, pages 87-92, July 2007.

[37] I. Li, A. K. Dey, and J. Forlizzi. Understanding my data, myself. Proceedings of UbiComp, page 405, 2011.

[38] J. J. Lin, L. Mamykina, S. Lindtner, G. Delajoux, and H. B. Strub. Fish'n'steps: encouraging physical activity with an interactive computer game. Proceedings of UbiComp, pages 261-278, 2006.

[39] G. Lupi and S. Posavec. Dear data, 2015 (accessed March 30, 2015). http://www.dear-data.com/.

[40] D. Mcadams. The stories we live by: personal myths and the making of self. The Guilford Press, 1993.

[41] M. Nunes, S. Greenberg, and C. Neustaedter. Using physical memorabilia as opportunities to move into collocated digital photo-sharing. International Journal of Human Computer Studies, 67:1087-1111, 2009.

[42] W. Odom, R. Banks, R. Harper, D. Kirk, S. Lindley, and A. Sellen. Tech- nology heirlooms ? considerations for passing down and inheriting digital materials. In Proceedings of CHI, pages 337-346, 2012.

[43] W. Odom, J. Zimmerman, and J. Forlizzi. Placelessness, spacelessness , and formlessness : experiential qualities of virtual possessions. In Proceedings of DIS, pages 985-994, 2014.

[44] H. Packer, A. Smith, and P. Lewis. Memorybook: generating narratives from lifelogs. In Proceedings of NHT, pages 7-11, 2012.

[45] D. Petrelli, S. Bowen, and S. Whittaker. Photo mementos: designing digital media to represent ourselves at home. International Journal of Human Computer Studies, 72(3):320-336, 2014.

[46] D. Petrelli and S. Whittaker. Family memories in the home: contrasting physical and digital mementos. Proceedings of UbiComp, 14(2):153$169,2010$.

[47] D. Petrelli, S. Whittaker, and J. Brockmeier. Autotopography: what can physical mementos tell us about digital memories? In Proceedings of CHI, pages 53-62, 2008.

[48] Z. Pousman, M. Romero, A. Smith, and M. Mateas. Living with tableau machine: a longitudinal investigation of a curious domestic intelligence. Proceedings of UbiComp, pages 370-379, 2008.

[49] Z. Pousman, J. Stasko, and M. Mateas. Casual information visualization: depictions of data in everyday life. TVCG, 13(6):1145-52, Jan. 2007.

[50] R. Sarvas and D. M. Frohlich. From snapshots to social media - the changing picture of domestic photography. Computer Supported Cooperative Work. Springer, 2011.

[51] A. Sellen, A. Fogg, M. Aitken, S. Hodges, C. Rother, and K. Wood. Do life-logging technologies support memory for the past? an experimental study using sensecam. Proceedings of CHI, pages 81-90, 2007.

[52] A. J. Sellen, S. Whittaker, and B. A. Sellen. Beyond total capture : a constructive critique of lifelogging. Communications of the ACM, 53:7077, 2010.

[53] C. Shen, N. Lesh, and F. Vernier. Personal digital historian: story sharing around the table. Interactions, 2003.

[54] M. M. Stevens, G. D. Abowd, K. N. Truong, and F. Vollmer. Getting intothe living memory box: family archives \& holistic design. Proceedings of UbiComp, 7:210-216, 2003.

[55] A. Stevenson. Oxford dictionary of english. Oxford Dictionary of English. OUP Oxford, 2010.

[56] S. Stusak, A. Tabard, F. Sauka, R. Khot, and A. Butz. Activity sculptures: exploring the impact of physical visualizations on running activity. TVCG, 20(12):2201-2210, 2014.

[57] A. Tat and S. Carpendale. Crystalchat : visualizing personal chat history. In Proceedings of HICSS, 2006.

[58] E. Thiry, S. Lindley, R. Banks, and T. Regan. Authoring personal histories: exploring the timeline as a framework for meaning making. Proceedings of CHI, pages 1619-1628, 2013.

[59] A. Thudt, D. Baur, and S. Carpendale. Visits : a spatiotemporal visualization of location histories. In EuroVis Short Papers, 2013.

[60] A. Thudt, D. Baur, and S. Carpendale. Autobiographical visualizations : challenges in personal storytelling. In DIS'14 Workshop on A Personal Perspective on Visualization and Visual Analytics, 2014.

[61] E. Tulving. Episodic and semantic memory. In Organization of Memory, pages 382-402. Academic Press, 1972.

[62] S. Turkle. Evocative objects: things we think with. MIT Press, 2007.

[63] E. van den Hoven and B. Eggen. Informing augmented memory system design through autobiographical memory theory. Proceedings of UbiCopm, 12:433-443, 2008.

[64] F. Viégas, danah Boyd, D. H. Nguyen, J. Potter, and J. Donath. Digital artifacts for remembering and storytelling : posthistory and social network fragments. In Proceedings of HICSS, page 40109.1, 2004.

[65] F. Viégas and J. Donath. Artifacts of the presence era: using information visualization to create an evocative souvenir. In IEEE Symposium on Information Visualization, 2002.

[66] F. Viégas, S. Golder, and J. Donath. Visualizing email content: portraying relationships from conversational histories. In Proceedings of $\mathrm{CHI}$, pages 979-988, 2006.

[67] Q. Wang and J. Brockmeier. Autobiographical remembering as cultural practice: understanding the interplay between memory, self and culture. Culture \& Psychology, 8:45-64, 2002.

[68] D. West, A. Quigley, and J. Kay. Memento: a digital-physical scrapbook for memory sharing. Proceedings of UbiComp, 11:313-328, 2007.

[69] R. Xiong and J. Donath. Peoplegarden: creating data portraits for users. In Proceedings of the Symposium on User Interface Software and Technology, pages $37-44,1999$. 